\title{
An approach to compare genome tiling microarray and MPSS sequencing data for transcript mapping Rajkumar Sasidharan*1,2, Ashish Agarwal ${ }^{1}$, Joel Rozowsky ${ }^{1}$ and Mark Gerstein ${ }^{1,3,4}$
}

Address: ${ }^{1}$ Molecular Biophysics and Biochemistry Department, Yale University, New Haven, CT 06520, USA, ${ }^{2}$ Department of Plant Biology, Carnegie Institution for Science, Stanford, California 94305, USA, ${ }^{3}$ Interdepartmental Program in Computational Biology and Bioinformatics, Yale University, New Haven, CT 06520, USA and ${ }^{4}$ Department of Computer Science, Yale University, New Haven, CT 06520, USA

Email: Rajkumar Sasidharan* - rsasidharan@stanford.edu; Ashish Agarwal - ashish.agarwal@yale.edu; Joel Rozowsky - joel.rozowsky@yale.edu; Mark Gerstein - mark.gerstein@yale.edu

* Corresponding author

Published: 21 October 2009

BMC Research Notes 2009, 2:21I doi:10.1186/1756-0500-2-2II
Received: 16 October 2009

Accepted: 21 October 2009

This article is available from: http://www.biomedcentral.com/l756-0500/2/2II

(c) 2009 Sasidharan et al; licensee BioMed Central Ltd.

This is an Open Access article distributed under the terms of the Creative Commons Attribution License (http://creativecommons.org/licenses/by/2.0), which permits unrestricted use, distribution, and reproduction in any medium, provided the original work is properly cited.

\section{Corrected abstract}

We are correcting the abstract of our published article ([I]). The sentence that starts "We observe that $4.5 \%$ of MPSS tags...." was not scientifically complete in the original abstract, having only two of the four numbers required to describe a comparison of two technologies in two different organisms. The abstract below more accurately describes our findings, as documented in Figure I of the manuscript.

\section{Background}

There are two main technologies for transcriptome profiling, namely, tiling microarrays and high-throughput sequencing. Recently there has been a tremendous amount of excitement about the latter because of the advent of next-generation sequencing technologies and its promises. Consequently, the question of the moment is how these two technologies compare. Here, we attempt to develop an approach to do a fair comparison of expressed transcripts identified from tiling microarray and MPSS tag sequencing data.

\section{Findings}

This comparison is a challenging task because the sequencing data is discrete while the tiling array data is continuous. We use the published Rice and Arabidopsis datasets which provide currently best matched sets of arrays and sequencing experiments using an earlier generation of sequencing technology, the MPSS tag sequencing approach. After scoring the arrays consistently in both the organisms, a first pass comparison reveals a surprisingly small overlap in expressed transcripts identified using the two technologies. We observe that $4.5 \%$ of MPSS tags overlap with $22 \%$ of transcripts detected from tiling array data in Rice while $13 \%$ of MPSS tags overlap with $66 \%$ of transcripts identified from tiling array data in Arabidopsis. However, a closer look at the data suggests that this is an underestimate. When we map tiling array probe intensities onto MPSS sequencing tags and then look at their intensity distribution, we see that the intensity distribution is very similar to exons detected from the respective tiling array data. Furthermore, restricting our comparison to only protein-coding gene loci reveals a very good overlap between the two technologies.

\section{Conclusion}

Our approach to compare genome tiling microarray and MPSS sequencing data suggests that there is actually a rea- 
sonable overlap in expressed transcripts identified by the two technologies. This overlap is distorted by the thresholding and scoring strategies employed in the tiling array transcript segmentation procedure.

\section{References}

I. Sasidharan R, Agarwal A, Rozowsky J, Gerstein M: An approach to compare genome tiling microarray and MPSS sequencing data for transcript mapping. BMC Research Notes 2009, 2:150.

Publish with Bio Med Central and every scientist can read your work free of charge

"BioMed Central will be the most significant development for disseminating the results of biomedical research in our lifetime. " Sir Paul Nurse, Cancer Research UK

Your research papers will be:

- available free of charge to the entire biomedical community

- peer reviewed and published immediately upon acceptance

- cited in PubMed and archived on PubMed Central

- yours - you keep the copyright

Submit your manuscript here:

http://www.biomedcentral.com/info/publishing_adv.asp 